\title{
A New Limit on Time-Reversal-Invariance Violation in Beta Decay: Results of the emiT-II Experiment
}

\author{
H. P. Mumm, ${ }^{* a}$ T. E. Chupp, ${ }^{b}$ K. P. Coulter ${ }^{b}$ R. L. Cooper, ${ }^{b}$ S. J. Freedman, ${ }^{c}$ \\ B. J. Fujikawa, ${ }^{c}$ A. García, ${ }^{d, e}$ G. L. Jones,${ }^{f}$ J. S. Nico, ${ }^{a}$ A. K. Thompson, ${ }^{a}$ C. A. Trull, ${ }^{g}$ \\ F. E. Wietfeldt ${ }^{g}$ and J. F. Wilkerson ${ }^{h}$ \\ ${ }^{a}$ National Institute of Standards and Technology, Gaithersburg, MD 20899, USA \\ ${ }^{b}$ Physics Department, University of Michigan, Ann Arbor, MI 48104, USA \\ ${ }^{c}$ University of California and Lawrence Berkeley National Lab, Berkeley, CA 94720, USA \\ ${ }^{d}$ CENPA and Physics Department, University of Washington, Seattle, WA 98195, USA \\ ${ }^{e}$ Department of Physics, University of Notre Dame, Notre Dame, IN 46556, USA \\ ${ }^{f}$ Department of Physics, Hamilton College Clinton, NY 13323, USA \\ ${ }^{g}$ Department of Physics, Tulane University, New Orleans, LA 70118, USA \\ ${ }^{h}$ Department of Physics, University of North Carolina, Chapel Hill, NC 27599, USA \\ E-mail: pieter@nist.gov
}

\begin{abstract}
We report on an improved determination of the parity-even, time-reversal-odd triple correlation, $\left.D<\vec{J}_{n}\right\rangle \cdot\left(\mathbf{p}_{\mathbf{e}} \times \mathbf{p}_{\mathbf{v}}\right)$, in the decay of cold polarized neutrons. Within the Standard Model, final state interactions give rise to $D \sim 10^{-5}$ and are calculable to better than $1 \%$ [1]. Thus, measurement of $D$ can provide a clear signal of time-reversal invariance violation and be used to limit extensions to the Standard Model. Our result is $D=(-0.96 \pm 1.89$ (stat $) \pm 1.01($ sys $)) \times 10^{-4}$. The corresponding phase between the axial vector and vector coupling constants is $\phi_{A V}=$ $180.013^{\circ} \pm 0.028^{\circ}$ ( $68 \%$ confidence level). This result represents the most sensitive measurement of $D$ in beta decay.
\end{abstract}

The 2011 Europhysics Conference on High Energy Physics, EPS-HEP 2011,

July 21-27, 2011

Grenoble, Rhône-Alpes, France

\footnotetext{
* Speaker.
} 


\section{Introduction}

The breaking of charge-parity $(\mathrm{CP})$ symmetry is necessary to explain the dominance of matter over antimatter in the early universe [2]. All observations of $\mathrm{CP}$ violation to date, for example in the $\mathrm{K}$ and $\mathrm{B}$ meson systems [3, 4], can be entirely accounted for by the single non-trivial phase in the Cabbibo-Kobayashi-Maskawa matrix in the electroweak Lagrangian. This phase, however, is known to be insufficient to account for the needed baryon asymmetry in the context of Big Bang cosmology [5]. We are thus given a compelling reason to search for new sources of CP violation in other systems. As CP and time-reversal (T) violation can be related to each other through the CPT theorem, experimental limits on electric dipole moments (T-odd) and T-odd observables in nuclear beta decay place strict constraints on some, but not all, possible sources of new $\mathrm{CP}$ violation. In the following we describe a test of time reversal symmetry in neutron beta decay.

\section{Measurement and Apparatus}

For nuclear beta decay, where the momentum transfer is negligible compared to the $\mathrm{W}$ masses, the most-general Lorentz-invariant Hamiltonian is [6]

$$
H_{\text {int }}=\sum_{i=V, A, S, T}\left(\bar{\psi}_{f} O_{i} \psi_{i}\right)\left(C_{i} \bar{\psi}_{v} O_{i} \psi_{e}+C_{i}^{\prime} \bar{\psi}_{v} \gamma_{5} O_{i} \psi_{e}\right) .
$$

where the left and right sides represent the hadronic and leptonic currents, respectively, and pseudoscalar contributions are assumed to be negligible in the nucleus. The operators $O_{i}$ are $O_{V}=\gamma_{\mu}$, $O_{A}=\gamma_{\mu} \gamma_{5}, O_{S}=1$, and $O_{T}=\sigma_{\mu v}$. In the Standard Model only $C_{V}=C_{V}^{\prime}$ and $C_{A}=C_{A}^{\prime}$ are nonzero. This Hamiltonian implies a probability distribution for neutron beta decay, $d W$, which can be written in terms of the polarization $\mathbf{P}$ and the momenta (energies) of the electron $\mathbf{p}_{e}\left(E_{e}\right)$ and antineutrino $\mathbf{p}_{v}\left(E_{v}\right)$ as [6]

$$
d W \propto 1+a \frac{\mathbf{p}_{e} \cdot \mathbf{p}_{v}}{E_{e} E_{v}}+b \frac{m_{e}}{E_{e}}+\mathbf{P} \cdot\left(A \frac{\mathbf{p}_{e}}{E_{e}}+B \frac{\mathbf{p}_{v}}{E_{v}}+D \frac{\mathbf{p}_{e} \times \mathbf{p}_{v}}{E_{e} E_{v}}\right) .
$$

A contribution of the parity-even triple correlation $D \mathbf{P} \cdot \mathbf{p}_{e} \times \mathbf{p}_{v}$ above the level of calculable finalstate interactions (FSI) implies T-violation. In the neutron rest frame, the triple correlation can be expressed as $D \mathbf{P} \cdot \mathbf{p}_{p} \times \mathbf{p}_{e}$, where $\mathbf{p}_{p}$ is the proton momentum. Thus one can extract $D$ from the spin dependence of proton-electron coincidences in the decay of cold polarized neutrons.

Our measurement was carried out at the National Institute of Standards and Technology Center for Neutron Research [7]. The detector, shown schematically in Fig. 1, consisted of an octagonal array of four electron-detection planes and four proton-detection planes concentric with a longitudinally polarized beam. The beam, with a neutron capture fluence rate at the detector of $1.7 \times 10^{8}$ $\mathrm{cm}^{-2} \mathrm{~s}^{-1}$, was defined using a series of $\mathrm{LiF}$ apertures and polarized to $>91 \%$ (95\% C.L.) by a double-sided bender-type supermirror [7]. A $560 \mu \mathrm{T}$ guide field maintained the polarization direction throughout the fiducial volume, while a current-sheet spin-flipper was used to reverse the neutron spin direction every $10 \mathrm{~s}$. The symmetric octagonal geometry was chosen to maximize sensitivity to $D$ while approximately canceling systematic effects stemming from detector efficiency variations or coupling to the spin-correlations $A$ and $B$ [8]. Each of the four proton segments consisted of a $2 \times 8$ array of silicon surface-barrier diode detectors (SBDs) with an active 

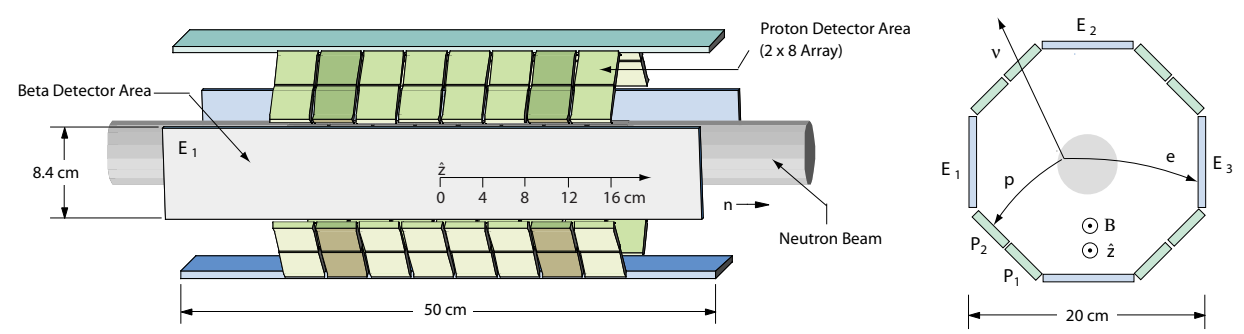

Figure 1: A schematic of the emiT detector illustrating the alternating electron and proton detector segments. The darker shaded proton detectors indicate the the paired-ring at $z= \pm 10 \mathrm{~cm}$. The cross section view illustrates, in a greatly exaggerated manner, the effect of the magnetic field on the particle trajectories and average opening angle. $\mathrm{A}_{2} \mathrm{E}_{3}$ coincidence event is shown.

layer $300 \mathrm{~mm}^{2} \times 300 \mu \mathrm{m}$. Each SBD was contained within an acceleration and focusing cell consisting of a $97 \%$ transmitting grounded wire-mesh box through which the recoil protons entered. Each SBD, situated within a field-shaping cylindrical tube, was held at a fixed voltage in the range $-25 \mathrm{kV}$ to $-32 \mathrm{kV}$. The sensitive regions of the beta detectors were plastic scintillator measuring $50 \mathrm{~cm}$ by $8.4 \mathrm{~cm}$ by $0.64 \mathrm{~cm}$ thick, with photomultiplier tube (PMTs) readout at both ends. This thickness is sufficient to stop electrons at the decay endpoint energy of $782 \mathrm{keV}$.

Data were acquired over a period from October 2002 to November 2003. Of these raw events, $12 \%$ were eliminated by filtering on various operational parameters (e.g. coil currents) and by requiring equal counting time in each spin-flip state. An additional 20\% were cut to enforce a uniform beta-energy software threshold of $90 \mathrm{keV}$ and by requiring beta multiplicity of one. The resulting data set contained more than $3 \times 10^{8}$ candidate coincidence events. These events were divided into two timing windows, a preprompt window from $-12.3 \mu$ s to $-0.75 \mu$ s that was used to determine the background from random coincidences and the decay window from $-0.5 \mu \mathrm{s}$ to $6.0 \mu \mathrm{s}$. The recoil proton with an endpoint of $750 \mathrm{eV}$ is, on average, delayed by $\sim 0.5 \mu \mathrm{s}$. The preprompt background energy spectrum was subtracted on a bin-by-bin basis from the proton spectrum in the decay window. The average signal-to-background was $\sim 30 / 1$. Finally, significant additional run time was used for calibrations and systematic studies.

Achieving the desired sensitivity to $D$ in the presence of the much larger spin-asymmetries due to $A$ and $B$ depends critically on the measurement symmetry. To extract $D$, coincident events are first combined into approximately efficiency-independent asymmetries

$$
w^{p_{i} e_{j}}=\frac{N_{+}^{p_{i} e_{j}}-N_{-}^{p_{i} e_{j}}}{N_{+}^{p_{i} e_{j}}+N_{-}^{p_{i} e_{j}}} \approx \mathbf{P} \cdot\left(A \tilde{\mathbf{K}}_{A}^{p_{i} e_{j}}+B \tilde{\mathbf{K}}_{B}^{p_{i} e_{j}}+D \tilde{\mathbf{K}}_{D}^{p_{i} e_{j}}\right),
$$

where $N_{+}^{p_{i} e_{j}}$ is the integrated number of coincident events in proton detector $p_{i}=1 \ldots 64$, beta detector $e_{j}=1 \ldots 4$, with neutron spin $+(-)$ aligned (anti-aligned) with the guide field. A constant unknown offset is added at this point to blind the analysis. The right-hand-side of Eqn. 2.3 assumes uniform polarization, $\mathbf{P}$. The K's are obtained from Eqn. 2.2 by integrating the normalized kinematic terms over the phase space of the decay, the neutron beam volume, and the acceptance of the indicated detectors [8]. Because of the geometry of the experiment, the terms stemming from the parity-violating $A$ and $B$ correlations can be nearly eliminated by further combining the data as 


$$
v^{p_{i}}=\frac{1}{2}\left(w^{p_{i} e_{R}}-w^{p_{i} e_{L}}\right),
$$

where $e_{R}$ and $e_{L}$ label the electron-detector at approximately $135^{\circ}$ giving a positive and negative cross-product $\mathbf{p}_{p} \times \mathbf{p}_{e}$ respectively; $\mathrm{P}_{2} \mathrm{E}_{3}$ vs $\mathrm{P}_{2} \mathrm{E}_{2}$ as shown in Fig. 1. Finally the values of $v^{p_{i}}$ are averaged over the sixteen proton-cells at the same $|z|$, i.e. $\pm 2, \pm 6, \pm 10$, and $\pm 14 \mathrm{~cm}$. Each set of detectors corresponds to paired-rings with the same symmetry as the full detector, e.g. the shaded detectors in Fig. 1. The experiment thus produces four independent measurements of $D$.

\section{Results}

The symmetry of the experiment is broken by a variety of effects which require a correction to the measured $D$. The largest are the following. High proton-detector energy thresholds lead to an error in the individual $w^{p_{i} e_{j}}$. Expansion of the neutron beam combined with the $560 \mu \mathrm{T}$ magnetic field affects the average of proton-electron angular correlation differently for upstream-downstream proton-cell pairs. Finally the azimuthal asymmetry of the neutron beam, most strongly influenced by the super-mirror polarizer, coupled with the transverse polarization of the neutron beam breaks the symmetry of the detector. Corrections were determined using a combination of calibration runs that magnified specific systematic effects and Monte Carlo simulations of the experiment. These are described in detail elsewhere [9]. The total of all systematic corrections is $-1.68 \pm 1.01$. Applying this correction we are left with the final result $D=(-0.96 \pm 1.89$ (stat $) \pm 1.01($ sys $)) \times 10^{-4}$. The corresponding upper limit on $D$ is a factor of three improvement over the previous upper limit for neutron decay $[8,10]$ as well as an improvement over that from ${ }^{19} \mathrm{Ne}$ decay [11], and thus this result represents the most sensitive test of time-reversal invariance in beta decay. Assuming only vector and axial vector interactions in beta decay, the result can be interpreted as a measure of the phase $\phi_{A V}=(180.013 \pm 0.028)^{\circ}$. Finally as can be seen from the generalized Hamiltonian in Eqn. 2.1, $D$ could arise for non-VA interactions, and this result constrains such contributions.

\section{References}

[1] S. Ando, J. McGovern, and T. Sato, Phys. Lett. B 667, 109 (2009).

[2] A. D. Sakharov, Sov. Phys. Usp. 34, 417 (1991).

[3] J. H. Christenson et al., Phys. Rev. Lett. 13, 138, (1964).

[4] K. Abe et al., Phys. Rev. Lett. 87, 091802, (2001).

[5] A. Riotto and M. Trodden, Ann. Rev. Nucl. Part. Sci. 49, 35, (1999).

[6] J. D. Jackson, S. B. Treiman, and H. W. Wyld Jr., Nucl. Phys. 4, 206 (1957).

[7] H. P. Mumm et al., Rev. Sci. Inst. 75, 5343 (2004)

[8] L. J. Lising et al., Phys. Rev. C 62, 055501 (2000).

[9] H. P. Mumm et al., Phys. Rev. Lett. 107, 102301 (2011).

[10] T. Soldner et al., Phys. Lett. B 58149 (2004).

[11] F. Calaprice et al., Hyperfine Interactions 22, 83, (1985). 